\title{
Roles of Different Forms of Lipopolysaccharides in Ralstonia solanacearum Pathogenesis
}

\author{
Chien-Hui Li, ${ }^{1}$ Kuan-Chung Wang, ${ }^{1}$ Yu-Hau Hong, ${ }^{1}$ Tai-Hsiang Chu, ${ }^{1}$ Yu-Ju Chu, ${ }^{1}$ I-Chun Chou, ${ }^{1}$ \\ Der-Kang Lu, ${ }^{1}$ Chiao-Yen Chen, ${ }^{1}$ Wen-Chieh Yang, ${ }^{1}$ Yu-Mei Lin, ${ }^{1}$ and Chiu-Ping Cheng ${ }^{1,2}$ \\ ${ }^{1}$ Graduate Institute of Plant Biology and ${ }^{2}$ Department of Life Science, College of Life Science, National Taiwan University, \\ Taipei, Taiwan, 106, Republic of China
}

Submitted 27 August 2013. Accepted 7 December 2013.

\begin{abstract}
Lipopolysaccharides (LPS) are critical components for the fitness of most gram-negative bacteria. Ralstonia solanacearum causes a deadly wilting disease in many crops; however, the pathogenic roles of different forms of LPS and their pathways of biogenesis remain unknown. By screening for phage-resistant mutants of $R$. solanacearum Pss4, whose genome sequence is unavailable, mutants with various types of structural defects in LPS were isolated. Pathogenesis assays of the mutants revealed that production of rough LPS (R-LPS), which does not contain O-polysaccharides, was sufficient to cause necrosis on Nicotiana benthamiana and induce the hypersensitive response on $N$. tabacum. However, biosynthesis of smooth LPS (S-LPS), which contains O-polysaccharides, was required for bacterial proliferation at infection sites on $N$. benthamiana leaves and for proliferation and causing wilt on tomato. Complementation tests confirmed the involvement of the previously unidentified cluster $R S c 2201$ to $R S c 2204$ in the formation of $R$. solanacearum S-LPS. With these data and the availability of the annotated genomic sequence of strain GMI1000, certain loci involved in key steps of $R$. solanacearum LPS biosynthesis were identified. The strategy of this work could be useful for similar studies in other bacteria without available genome sequences.
\end{abstract}

The soilborne bacterium Ralstonia solanacearum causes a destructive wilting disease in a wide range of plants, including many economically important crops such as tomato, potato, and banana. $R$. solanacearum invades plants through natural openings and wounds in roots. It colonizes and propagates in the xylem and secretes large quantities of extracellular polysaccharides, eventually leading to plant wilting and death (Denny 2006). To establish a successful systemic infection, $R$. solanacearum must overcome various stress conditions in soil and inside host plants.

The outer membrane (OM) of a gram-negative bacterium is an effective permeability barrier that protects cells from

C.-H. Li, K.-C. Wang, Y.-H. Hong, and T.-H. Chu contributed equally to this work.

Corresponding author: C.-P. Cheng; Telephone: +886-2-3366-3793; Fax +886-2-2391-8940; E-mail: chiupingcheng@ntu.edu.tw

* The $\boldsymbol{e}$-Xtra logo stands for "electronic extra" and indicates that four supplementary figures and four supplementary tables are published online.

(C) 2014 The American Phytopathological Society harmful compounds and, thus, allows bacterial survival in these hostile environments. The permeability features of the $\mathrm{OM}$ are mostly contributed by lipopolysaccharides (LPS), critical components of most gram-negative bacteria (Raetz and Whitfifeld 2002). Smooth LPS (S-LPS) are composed of a hydrophobic membrane-anchored lipid A moiety, a short core oligosaccharide, and a distal and more variable $\mathrm{O}$-antigen polysaccharide chain. LPS not containing O-antigen are referred to as core-lipid A or rough LPS (R-LPS). The synthesis and assembly of these components have been well documented in Escherichia coli (Raetz and Whitfifeld 2002; Wang and Quinn 2010). Briefly, the first step in LPS synthesis involves synthesis of lipid A from uridine diphosphate $\mathrm{N}$ acetylglucosamine via a conserved pathway, which takes place in the cytoplasm and is engaged by proteins on the cytoplasmic surface of the inner membrane (IM). This process is accomplished by a group of Lpx proteins and KdtA. Lipid A then serves as the acceptor for the assembly of the core oligosaccharide via sequential glycosyl transfer triggered by the action of GmhD and a group of Waa glycosyltransferases, leading to the formation of R-LPS. The glycosyltransferases and polymerases encoded by the $r f b$ gene cluster are required for synthesis and transfer of $\mathrm{O}$-antigen polysaccharide precursors across the IM and then $\mathrm{O}$-antigen polymerization is promoted by Wzy and $\mathrm{Wzz}$ on the periplasmic face of the IM. MsbA, an essential ATP-binding cassette transporter, flips the R-LPS across the IM where ligation of $\mathrm{O}$-antigen to R-LPS is then triggered by WaaL to form SLPS, which is finally transported to the OM (Chng et al. 2010a and b; Sperandeo et al. 2009). The structures of lipid $\mathrm{A}$ and the core are largely conserved among diverse bacteria, while the $\mathrm{O}$-antigens display significant structural diversity (Wilkinson 1996). However, O-antigen structures are mostly uniform in some strains of bacteria, including $R$. solanacearum (Kocharova et al. 1993).

In addition to providing a barrier to various harsh environments, LPS serve critical roles in microbe-plant interactions by promoting biofilm formation, host attachment, and colonization; allowing bacterial growth and survival in unfavorable niches on and within hosts; modulating host defense; responses and altering the host range (Dow et al. 2000; Newman et al. 2007; Ormeno-Orrillo et al. 2008). A role for LPS in suppressing the host hypersensitive response (HR), a type of rapid cell death in the local region near infection sites to prevent the spread of pathogens, was initially established for $R$. solanacearum (Graham et al. 1977). Although the composition of $R$. solanacearum LPS has been studied extensively (Kocharova et al. 1993; Varbanets et al. 2003; Whatley et al. 1980; Zdorovenko et al. 2008), the determinants involved in 
LPS biosynthesis and the pathogenic roles of its different LPS forms remain to be investigated. Prior to the present study, only one gene $(r f a F / R S c 0565)$ involved in the production of the LPS core had been identified and its disruption led to avirulence (Titarenko et al. 1997).

LPS of gram-negative bacteria are known to be required for phage adsorption (Michel et al. 2010; Prehm et al. 1976; Qimron et al. 2006). To identify $R$. solanacearum genes functioning in LPS biogenesis and determine the roles of specific LPS forms in bacterial pathogenesis, we screened transposoninsertional mutants in strain Pss4 (phylotype I, biovar 3) (Lin et al. 2008) for resistance to a phage (RS $\varphi$ P29) isolated in Taiwan (unpublished data). Because production of intact S-LPS was found to be crucial for the adsorption of $\mathrm{RS} \varphi \mathrm{P} 29$ (unpublished data), this phage-resistance-based screen led to the isolation of mutants with various types of structural defects in LPS. Characterization of the mutants showed that R-LPS production is important for triggering necrosis on Nicotiana benthamiana and inducing HR on N. tabacum. On the other hand, full bacterial virulence, including bacterial proliferation at infection sites on $N$. benthamiana leaves and proliferation and wilt induction on tomato, requires S-LPS biosynthesis. Although the genome of strain Pss4 has not yet been sequenced, a brief LPS biosynthetic pathway of $R$. solanacearum was proposed by analyzing the identified genes based on the annotated genomic sequence of strain GMI1000. Our strategy could be used to identify genes required for LPS biogenesis in other bacteria without available genome sequences.

\section{RESULTS}

Identification of disrupted loci

in $\boldsymbol{R}$. solanacearum phage-resistant mutants.

A Podovirus T7-like phage, $\mathrm{RS} \varphi \mathrm{P} 29$, which is able to completely lyse the wild-type $R$. solanacearum Pss4, failed to infect the $R$. solanacearum rfaF (RSc0565) mutant (unpublished data), suggesting that the phage could be used to screen for
LPS-defective bacterial mutants. An $R$. solanacearum Pss4 random transposon (Tn5)-insertion library containing 24,400 mutants was constructed. Eighty-two of the mutants reproducibly survived in the presence of RS $\varphi \mathrm{P} 29$. The sequences flanking the transposon insertion sites in 52 of the mutants were successfully determined by thermal asymmetrical interlaced (TAIL) polymerase chain reaction (PCR) and confirmed by genomic PCR (Table 1). Comparison of these sequences with the genome sequence of $R$. solanacearum GMI1000 indicated that 19 different genes were inserted by $\operatorname{Tn} 5$, in several cases at multiple independent sites (Table 1). The identification of three independent insertions for RSc0565/rfaF confirmed the effectiveness of our screens for mutants resistant to RS $\varphi$ P29. Seventeen of the Tn5-inserted genes are located in clusters of two or more adjacent genes with related functions (Table 1; Supplementary Fig. S1). Among the 19 genes, five ( $R S c 0692$, $R S c 0914, R S c 0915, R S c 2204$, and $R S c 0565)$ are orthologs of genes involved in LPS biogenesis, two (RSc0691 and RSc3058) are likely involved in mannose metabolism, six ( $R S c 0688$, $R S c 0689, R S c 2201, R S c 2202, R S c 2203$, and $R S c 0523)$ are orthologous to proteins involved in glycosylation and related modifications, four (RSc2958, RSc2959, RSc2960, and $R S c 2962)$ are $\mathrm{ABC}$ transporter or membrane proteins, and two $(R S c 3059, R S c 0154)$ are likely to be involved in sugar or muropeptide transport.

\section{LPS production.}

The overall growth of all of the RS $\varphi \mathrm{P} 29$-resistant mutants in the rich agar medium CPG agar medium (Denny and Hayward 2001) or in minimal liquid medium M9 was not significantly different from that of the wild-type strain (data not shown). The pattern of LPS production by the mutants was further examined by sodium dodecyl sulfate (SDS) polyacrylamide gel electrophoresis and silver staining (Fig. 1; Supplementary Fig. $\mathrm{S} 2$; Table 2). To better illustrate profiles of LPS production, three of the Tn5 mutants obtained were used as a reference. These included mutants of RSc0914 (rfaE) and RSc0915

Table 1. Phage-resistant Ralstonia solanacearum mutants identified in this study and the tagged loci

\begin{tabular}{|c|c|c|c|}
\hline Clusters or loci identified & $\begin{array}{c}\text { Transposon } \\
\text { insertion sites }\end{array}$ & $N^{\mathrm{a}}$ & Putative Escherichia coli ortholog ${ }^{\mathbf{b}}$ \\
\hline \multicolumn{4}{|l|}{ Clusters } \\
\hline \multirow[t]{5}{*}{ RSc0688-0693 } & $R S c 0688$ & 9 & Glycosyl transferase \\
\hline & $R S c 0689$ & 3 & Putative undecaprenyl phosphate $\mathrm{N}$-acetylglucosaminyltransferase transmembrane protein \\
\hline & $R S c 0691$ & 1 & Putative phosphomannomutase (PMM) or phosphoglucomutase \\
\hline & $R S c 0692(r f a C 1)$ & 1 & Lipopolysaccharide heptosyltransferase $\mathrm{RfaC} 1$ \\
\hline & $R S c 0693(k d t A)^{\mathrm{c}}$ & 0 & 3-Deoxy-D-manno-octulosonic-acid transferase KdtA \\
\hline \multirow[t]{2}{*}{$R S c 0914-0915$} & $R S c 0914(r f a E)$ & 1 & ADP-heptose synthase RfaE \\
\hline & $R S c 0915(r f a D)$ & 2 & ADP-L-glycero-D-manno-heptose-6-epimerase RfaD \\
\hline \multirow[t]{5}{*}{$R S c 2200-2204$} & $R S c 2200^{\circ}(m s b A)$ & 0 & Lipid A export ABC transporter ATP-binging protein MsbA \\
\hline & $R S c 2201$ & 2 & Putative signal peptide protein (glycosyl transferase family 2) \\
\hline & $R S c 2202(I g t F)$ & 3 & Glycosyl transferase \\
\hline & $R S c 2203$ & 4 & Hypothetical protein (glycosyl transferase family 2) \\
\hline & $R S c 2204$ & 3 & Probable lipopolysaccharide $\mathrm{O}$-antigen ligase transmembrane protein \\
\hline \multirow[t]{4}{*}{$R S c 2958-2962$} & $R S c 2958$ & 4 & Putative ABC-type toluene transporter \\
\hline & $R S c 2959$ (vacJ) & 2 & Lipoprotein precursor $(\mathrm{VACJ})$; transmembrane protein \\
\hline & $R S c 2960$ & 1 & Putative ABC-type transporter \\
\hline & $R S c 2962$ & 1 & $\mathrm{ABC}$ transporter ATP-binding protein \\
\hline \multirow[t]{2}{*}{ RSc3058-3059 } & $\operatorname{RSc} 3058(\operatorname{man} A)$ & 2 & Mannose-6-phosphate isomerase \\
\hline & $R S c 3059$ & 1 & Sugar transporter transmembrane protein \\
\hline \multicolumn{4}{|l|}{ Single loci } \\
\hline$R S c 0154$ & $R S c 0154(\operatorname{amp} G)$ & 6 & Muropeptide transporter \\
\hline$R S c 0523$ & $R S c 0523$ & 3 & D,D-heptose 1,7-bisphosphate phosphatase \\
\hline$R S c 0565$ & $R S c 0565(r f a F)$ & 3 & ADP-heptose-lipopolysaccharide heptosyltransferase II RfaF \\
\hline
\end{tabular}

${ }^{a}$ Number of independent mutants with transposon insertions at different locations within each gene.

${ }^{\mathrm{b}}$ Gene sequences of strain GMI1000 were used to retrieve orthologous sequences from the National Center for Biotechnology Information and KEGG databases. Putative E. coli orthologs sharing sequence similarity $\geq 20 \%$ with each gene were identified by PSI and PHI-BLAST.

${ }^{c}$ Mutations in $R S c 0693$ and $R S c 2200$ were not identified in this study but these genes are orthologous to $k d t A$ and $m s b A$, respectively, and considered to potentially contribute to the defective phenotypes in phage resistance and LPS production due to possible polar effects of Tn5 insertions in $R S c 0692$ and RSc2201. 
$(r f a D)$, whose orthologs in enteric bacteria specify the synthesis and addition of heptose to the LPS core to produce R-LPS, and a mutant of $R S c 0565$ ( $r f a F)$, a gene involved in the production of the LPS core in $R$. solanacearum (Titarenko et al. 1997). R-LPS was absent in the wild-type strain (Figs. 1 and 2A), except for a few cases due to unknown reasons. As expected, the three reference mutants produced only low molecular weight molecules, which could be the expected $\mathrm{Kdo}_{2}$-lipid, but failed to synthesize R- and S-LPS. Mutants containing Tn5 insertions in $R S c 0692, R S c 0523, R S c 3058$, and $R S c 3059$ were unable to produce R- and S-LPS. Mutants containing Tn 5 insertions in seven other genes (RSc0688, RSc0689, RSc2201, $R S c 2202, R S c 2203, R S c 2204$, and $R S c 0691)$ produced only RLPS or R-LPS with very limited O-antigen ligation, suggesting malfunctions in O-antigen polymerization or addition and, thus, S-LPS production. Mutant $X 1 \mathrm{~m}$ was unable to produce $\mathrm{R}$ - and S-LPS and $X 2 \mathrm{~m}$ produced only R-LPS. In addition, five of the mutants (RSc2958, RSc2959, RSc2960, RSc2962, and $R S c 0154)$ displayed an LPS production profile similar to that of the wild-type strain (Fig. 3; Table 2; data not shown).

\section{Pathogenesis assays.}

To investigate the roles of different forms of LPS in $R$. solanacearum pathogenesis, we carried out different assays. To determine pathogenicity of the LPS-defective mutants, the responses of two tobacco species to bacterial infection were examined. The wild-type Pss 4 strain caused necrosis on $N$. benthamiana leaves $72 \mathrm{~h}$ postinfiltration and an HR on $N$. tabacum W38 leaves $24 \mathrm{~h}$ postinfiltration (Table 2; Supplementary Fig. S3), reflecting normal virulence and indicating full function of the type III secretion system (T3SS), respectively. On $N$. benthamiana, mutants that were unable to produce R- and S-LPS (RSc0692, RSc0523, RSc0565, RSc0914, $R S c 0915, R S c 3058, R S c 3059$, and X1) failed to cause detectable disease symptom after direct introduction into leaves at a high titer (optical density at $600 \mathrm{~nm}\left[\mathrm{OD}_{600}\right]=0.3$ or approximately $10^{8} \mathrm{CFU} / \mathrm{ml}$ ) (Table 2). However, mutants that produced only R-LPS (RSc0688, RSc0689, RSc2201, RSc2202,
$R S c 2203, R S c 2204, R S c 0691$, and X2) caused normal or slightly reduced necrotic symptoms. These data revealed that R-LPS production was sufficient to cause necrosis on $N$. benthamiana. However, in planta assays showed that the prolifera-

Table 2. Lipopolysaccharide (LPS) pattern and plant responses

\begin{tabular}{|c|c|c|c|}
\hline \multirow[b]{2}{*}{ Transposon insertion loci } & \multirow[b]{2}{*}{ LPS pattern ${ }^{b}$} & \multicolumn{2}{|c|}{ Pathogenesis $^{\mathrm{a}}$} \\
\hline & & N. benth & N. tab \\
\hline Wild-type & S-LPS, R-LPS & $\mathrm{N}$ & HR \\
\hline \multicolumn{4}{|l|}{ LPS lipid A biogenesis } \\
\hline$R S c 0692(r f a C 1)$ & - & - & - \\
\hline \multicolumn{4}{|l|}{ LPS core biogenesis } \\
\hline$R S c 0523$ & - & - & - \\
\hline$R S c 0565(r f a F)$ & - & - & HR \\
\hline$R S c 0914(r f a E)$ & - & - & - \\
\hline$R S c 0915(r f a D)$ & - & - & HR \\
\hline \multicolumn{4}{|l|}{ LPS O-antigen biogenesis } \\
\hline$R S c 0688$ & R-LPS & $\mathrm{S}$ & HR \\
\hline$R S c 0689$ & R-LPS & $\mathrm{N}$ & HR \\
\hline$R S c 2201$ & R-LPS & $\mathrm{N}$ & HR \\
\hline$R S c 2202($ IgtF $)$ & R-LPS & $\mathrm{N}$ & HR \\
\hline$R S c 2203$ & R-LPS & $\mathrm{N}$ & HR \\
\hline \multicolumn{4}{|l|}{ LPS O-antigen ligation } \\
\hline$R S c 2204$ & R-LPS & $\mathrm{N}$ & HR \\
\hline \multicolumn{4}{|l|}{ Mannose metabolism } \\
\hline$R S c 0691$ & R-LPS & $\mathrm{S}$ & HR \\
\hline$R S c 3058(\operatorname{manA})$ & - & - & - \\
\hline \multicolumn{4}{|l|}{ Sugar transport } \\
\hline$R S c 3059$ & - & - & - \\
\hline \multicolumn{4}{|l|}{ Unidentified loci } \\
\hline$X 1$ & - & - & - \\
\hline$X 2$ & R-LPS & $\mathrm{N}$ & HR \\
\hline
\end{tabular}

a The response of Nicotiana benthamiana (N. benth) and $N$. tabacum $(N$. $t a b)$ W38 was examined 72 and $24 \mathrm{~h}$, respectively, after inoculation with bacteria by leaf infiltration. $\mathrm{N}=$ necrosis, $\mathrm{S}=$ slight necrosis, $\mathrm{HR}=$ hypersensitive response, $-=$ no response. The experiment was repeated at least three times with consistent results.

b The experiment was performed on at least two independent bacterial colonies and repeated at least three times using independent biological samples with consistent results. S-LPS = smooth LPS, R-LPS = only rough (R)-LPS is produced, $-=$ neither S-LPS nor R-LPS is produced.
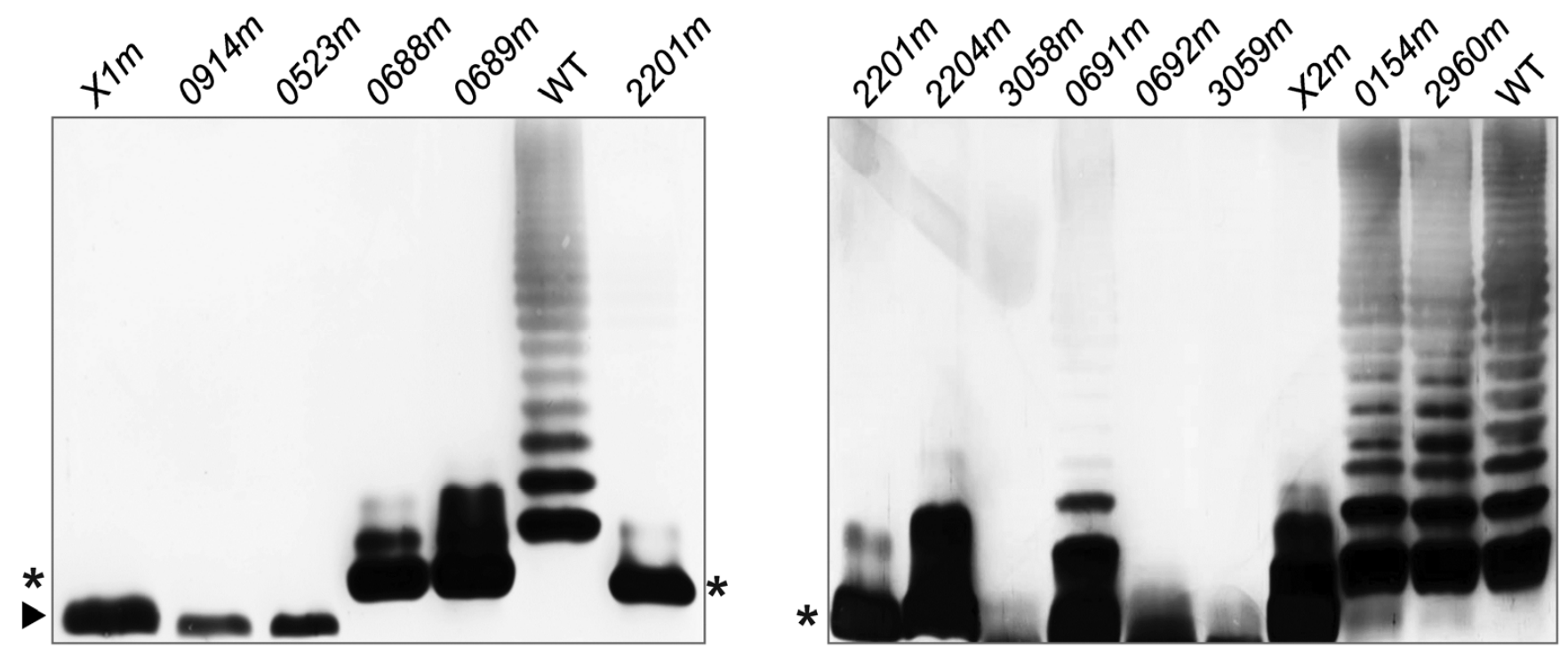

Fig. 1. Lipopolysaccharide (LPS) profiles of representative Ralstonia solanacearum mutants. LPS specimens extracted from the indicated strains were separated on 4 to $12 \%$ sodium dodecyl sulfate-polyacrylamide gel and stained with silver stain. The experiment was performed on at least three independent bacterial colonies and repeated at least three times using independent biological samples with consistent results. Results from a single representative trial are presented. WT $=$ the wild-type Pss 4 strain. Mutants are indicated by their Tn5-inserted loci; for example, the mutant carrying Tn5 in RSc0914 is indicated as $0914 \mathrm{~m}$. Two mutants carrying Tn5 in unidentified loci are indicated as $X 1 \mathrm{~m}$ and $X 2 \mathrm{~m}$. A mutant of $R S c 0914 / r f a E(0914 \mathrm{~m})$, whose homologs in enteric bacteria are involved in rough (R)-LPS biosynthesis, was unable to produce R-LPS and smooth LPS and, thus, was used as a reference strain for the analysis. A mutant of $R S c 2201(2201 \mathrm{~m})$, which produced only R-LPS, was included in both gels as another reference strain. Stars indicate R-LPS; the arrowhead indicates the expected $\mathrm{Kdo}_{2}$-lipid. 
tion of LPS-defective mutants in leaves after direct introduction into $N$. benthamiana leaves at a low titer (approximately $10^{4}$ to $10^{5} \mathrm{CFU} / \mathrm{ml}$ ) was severely impaired (Supplementary Fig. S4), indicating the requirement of S-LPS production for achieving a wild-type level of in planta bacterial propagation. On N. tabacum, mutants that produced only R-LPS could induce HR similarly to the wild-type strain, whereas mutants unable to produce R- and S-LPS mostly failed to induce HR, except for the $R S c 0565$ and $R S c 0915$ mutants (Table 2). These results suggested a role of R-LPS in T3SS function.

To further investigate whether the LPS-defective mutants were defective in establishing a systemic infection in a natural pathosystem, a virulence assay on the susceptible tomato 'L390' was carried out by soil drench without root severing. The wildtype strain caused severe wilting on most plants approximately 10 to 12 days postinoculation, whereas all of the LPS-defective mutants were unable to produce any disease symptom throughout the period of our evaluation (Fig. 3A) or only caused slight wilting on very few plants (Fig. 3B). Consistently, bacterial populations of two mutants, $R S c 0565$ (unable to produce Rand S-LPS) and RSc0688 (able to produce only R-LPS) in the stem-base of tomato L390 were less than $10^{3} \mathrm{CFU} / \mathrm{g}$ of fresh tissue (data not shown). These results revealed the importance of S-LPS production in achieving successful systemic infection in the host plant.
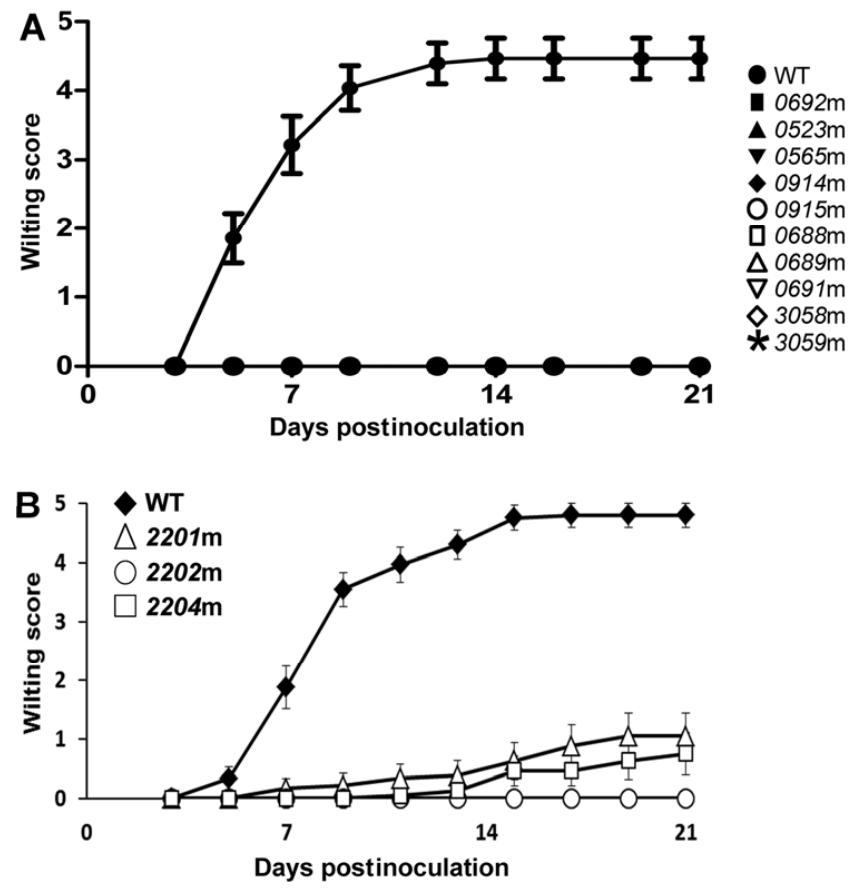

Fig. 2. Complementation tests of the RSc2201 to -2204 mutants. A, Lipopolysaccharide (LPS) profile resolved on a $16 \%$ tricine-sodium dodecyl sulfate-polyacrylamide gel and stained with silver stain. The experiment was performed on at least three independent bacterial colonies and repeated three times using independent biological samples with consistent results. Results from a single representative sample are presented. Stars indicate rough (R)-LPS; arrowheads indicate the expected $\mathrm{Kdo}_{2}$-lipid. $\mathbf{B}$ to E, Virulence test on tomato L390 by soil drench without root severing. The test was carried out on 143 -week-old plants per strain in each trial and repeated independently twice with consistent results. Results presented are the average of 28 plants from two independent trials, with the error bars denoting the standard deviations. The RSc0565/rfaF mutant $(0565 \mathrm{~m})$, which is unable to produce R-LPS and smooth LPS, was used as a reference strain. WT $=$ the wild-type Pss 4 strain; $2201 \mathrm{~m}=$ the $R S c 2201$ mutant; $2202 \mathrm{~m}=$ the $R S c 2202$ mutant; $2204 \mathrm{~m}=$ the RSc2204 mutant. For complementation test, recombinant strains carrying the empty vector or vectors containing the expressed genes are labeled with $\mathrm{V}$ or the names of the expressed genes, respectively.
Complementation tests

with the $R S c 2201$ to $R S c 2204$ mutants.

The $R S c 2204$ gene product is a putative ortholog of E. coli WaaL, which triggers the ligation of O-antigen to R-LPS to form S-LPS, and the involvement of cluster RSc2201 to $R S c 2203$ and their orthologs in LPS biogenesis has not been reported. Therefore, we further carried out transcomplementation tests. The results showed that $R S c 2201, R S c 2202$, and $R S c 2204$ mutants produced only R-LPS (Fig. 2A). Expression of a wild-type version of the genes in the corresponding mutant rescued the defects in LPS production and the virulence on susceptible tomato plants (Fig. 2B through E). These results confirmed that these genes are required for the formation of $R$. solanacearum S-LPS.

Sequence analysis of the identified $R$. solanacearum genes.

To further determine whether the LPS biosynthesis-related genes identified by our mutant screens and their flanking genes were conserved among gram-negative bacteria, comparative sequence analysis was carried out on representative bacteria (Supplementary Table S1). KdtA is involved in lipid A biosynthesis and its orthologs, including RSc0693, share a significant sequence similarity of at least $50 \%$. Proteins presumably participating in the biosynthesis of core oligosaccharides (RSc0523, RSc0565, RSc0914, and RSc0915) largely shared significant but varying degrees of similarity $(\geq 47 \%)$. For proteins probably involved in O-antigen biosynthesis, RSc0688 and RSc0689 and RSc2201 and RSc2203 mostly shared low sequence similarities, while orthologs of RSc0692 were relatively conserved (similarity $\geq 42 \%$ ). WaaL (RSc2204) orthologous proteins generally shared a sequence similarity of at least $30 \%$. Mannose metabolism-related proteins (RSc0691/ManB and RSc3058/ManA) and the putative sugar transporter RSc3059 were fairly conserved in the analyzed bacteria (similarity $\geq 48 \%$ ), except for a few putative RSc3058/ManA orthologs.

\section{DISCUSSION}

To successfully cause wilting in host plants, $R$. solanacearum has to sustain in various harsh environments throughout its systemic infection initiated from roots. Nevertheless, how LPS contributes to its pathogenesis and determinants of its LPS biogenesis pathway are almost undetermined. Two previous reports used phages to search for $R$. solanacearum LPS biosynthesis-defective mutants (Hendrick and Sequeira 1984; Whatley et al. 1980); however, the loci disrupted in the identified mutants remained undefined. In this study, we successfully used phage-resistance screening to isolate mutants with various types of LPS defects. In addition, with the availability of the annotated genome sequence of the closely related strain GMI1000, we were able to analyze possible functions of the identified targeted loci. The isolation of mutants with Tn5 insertions in orthologs of well-known LPS biogenesis-related genes (Table 1) confirmed the effectiveness of our screens.

Previously, an $R$. solanacearum K60 rfaF mutant defective in LPS production was shown to be avirulent (Titarenko et al. 1997). By characterizing mutants with various types of defects in LPS isolated from this study, our study points to a critical role for R-LPS in $R$. solanacearum T3SS function and in the virulence on $N$. benthamiana leaves after direct introduction at a high titer and (Table 2). However, R-LPS production was not adequate to sustain the wild-type level of in planta bacterial propagation at infection sites on $N$. benthamiana leaves after direct introduction at a low titer. In addition, R-LPS biosynthesis was not sufficient for proliferating and causing wilt on tomato (Fig. 3). Therefore, production of S-LPS is required for full virulence of $R$. solanacearum in the course of systemic infec- 
tion, particularly for effective bacterial proliferation in the stressful environment inside plants.

$R$. solanacearum LPS typically consists of two L-glyceroD-manno-heptose moieties (core) linking $\mathrm{Kdo}_{2}$-lipid A and O-antigen; in contrast with $E$. coli, the outer core structure contains mostly rhamnose rather than glucose or galactose (Zdorovenko et al. 2008). Although most of the genes involved in biosynthesis of LPS lipid A and inner core are conserved among diverse bacteria, $R$. solanacearum genes involved in core modification and outer core and $\mathrm{O}$-antigen biosynthesis are distinct from those found in E. coli. In addition, $R$. solanacearum LPS biosynthesis-related genes are clustered less compactly in the genome compared with those in E. coli. Therefore, in silico approaches would be insufficient to uncover determinants specific to $R$. solanacearum LPS biosynthesis. In this study, in addition to the well-conserved pro-

A

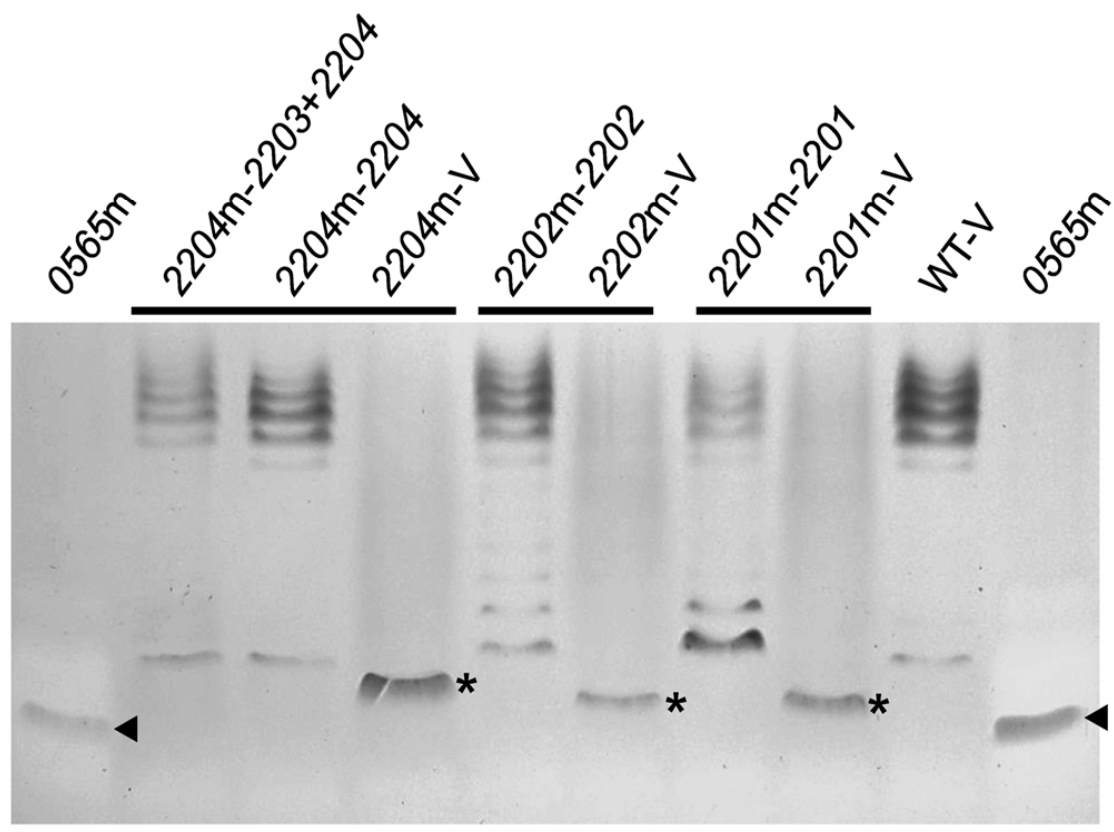

B

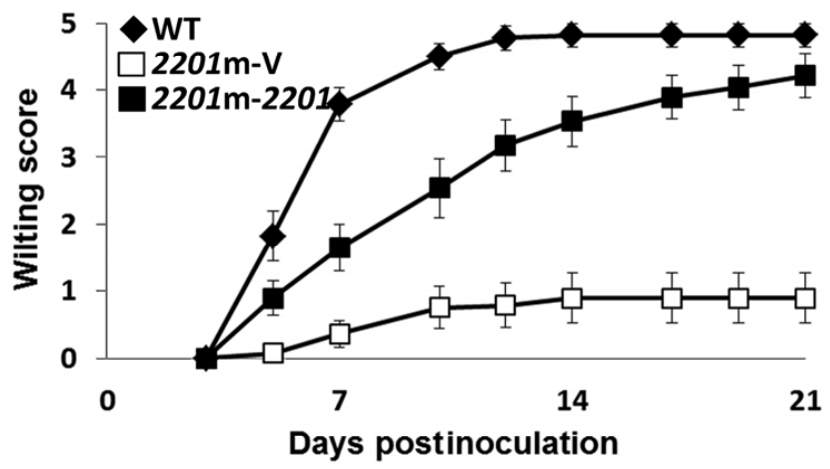

D

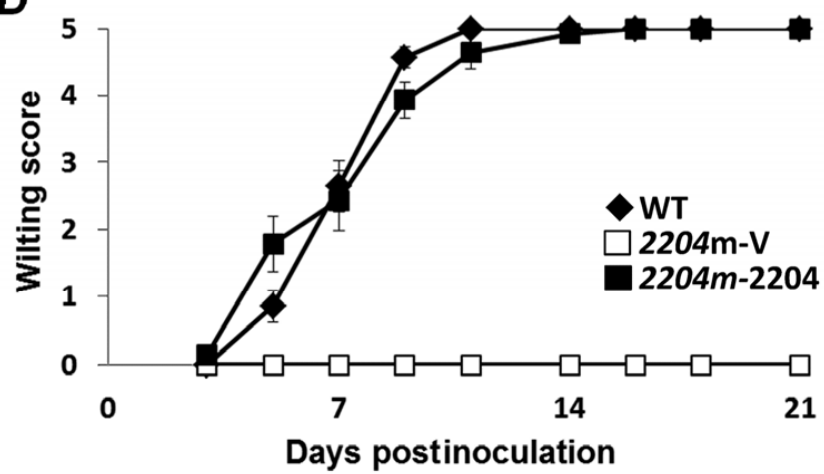

C

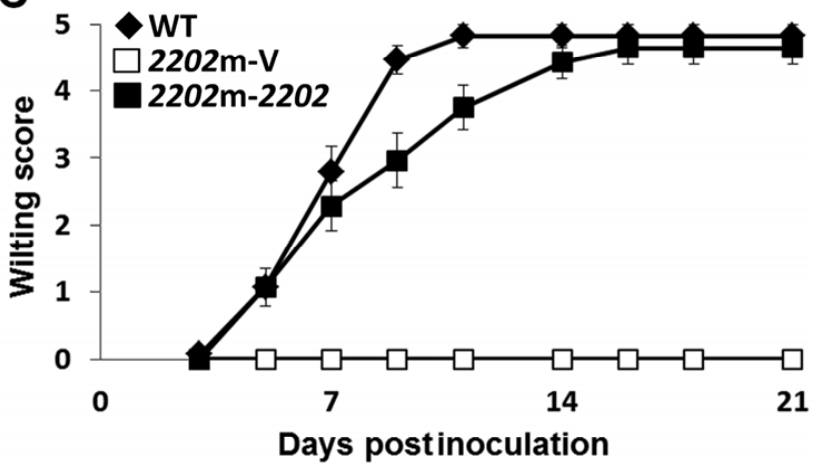

$\mathbf{E}$

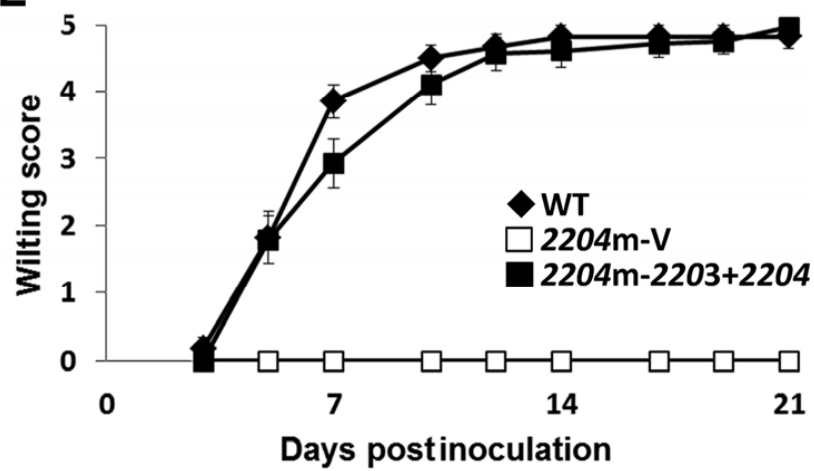

Fig. 3. Virulence test of Ralstonia solanacearum mutants on susceptible tomato L390. The experiment was carried out by soil drench without root severing. A, Nine 3-week-old plants were used for each strain in every trial and the test was repeated independently three times with consistent results. Results presented are the average of the data collected from three independent batches with the error bars denoting the standard deviations (SD). B, The test was carried out on 14 3-week-old plants per strain in each trial and repeated independently twice with consistent results. Results presented are the average of 28 plants from two independent trials with the error bars denoting the SD. WT = the wild-type Pss 4 strain. Mutants are indicated by their Tn5-inserted loci. 
teins involved in LPS biosynthesis (Table 1; Fig. 4), our screen also led to the identification of LPS biosynthesis-related loci that were not identified by in silico approaches (Fig. 4). For instance, our study evidences crucial roles for $R S c 2201$ to $R S c 2204$ and $R S c 0688$ to $R S c 0690$ in O-antigen polymerization or addition onto R-LPS to form S-LPS (Figs. 1 and 2).

The isolation and characterization of mutants with various structural defects in LPS, along with comparative sequence analyses of the Tn5-inserted loci and their flanking genes, allowed identification of loci involved in key steps in the $R$. solanacearum LPS biosynthesis pathway (Fig. 4). The conserved protein $\mathrm{RSc} 0693$ (KdtA) is projected to be involved in $\mathrm{Kdo}_{2}$-lipid A biosynthesis (step 1). RSc0914 (RfaE), $\mathrm{RSc} 0915$ ( $\mathrm{RfaD}$ ), and $\mathrm{RSc0523}(\mathrm{GmhB})$ participate in the biogenesis of core oligosaccharides (step 2). RSc0565 (RfaF) and $\mathrm{RSc0692}$ (WaaC/RfaC) are required for linking lipid A and the core to produce R-LPS (step 3). The biogenesis of Oantigen polysaccharide requires functional clusters RSc0688 to RSc0690 and RSc2201 to RSc2203 (step 4). RSc3058 and RSc0691 respectively share sequence homology with the mannose-6-phosphate isomerase ManA and the phosphomannomutase ManB. These enzymes are sequentially involved in the production of GDP-mannose, which is important for the biosynthesis of $\mathrm{O}$-antigen polysaccharide, and their mutations led to failure in S-LPS production (Collins et al. 1991; Heller and Braun 1982; Kim et al. 2003; Paixão et al. 2009). Consistently, mutation in R. solanacearum RSc0691 impaired S-LPS production. However, the RSc3058 mutants were defective in both $\mathrm{R}$ - and S-LPS production. Because the RSc3059 mutant also failed to produce R- and S-LPS, suggesting an unreported novel important function of the encoded probable sugar transporter for LPS biogenesis, the severe LPS defect of the $R S c 3058$ mutants might be due to a possible polar effect of the mutation on RSc3059. The conserved protein MsbA (RSc2200) is expected to flip the R-LPS across the IM, where WaaL (RSc2204) promotes ligation of O-antigen to R-LPS, leading to the formation of S-LPS (step $5)$. Because LPS structures are uniform in a large group of strains of $R$. solanacearum (Kocharova et al. 1993), the information established here might, for the most part, be universal among strains of this species complex.

Although the library pool size of our screens covered almost four times the expected number of $R$. solanacearum genes, the current screen approach is still insufficient to uncover many of the genes involved in LPS biosynthesis. This is because certain genes involved in LPS $\mathrm{Kdo}_{2}$-lipid A biosynthesis may be indispensable for bacterial viability, making inclusion of such genes in our mutant pools impossible. In addition, transposon insertion bias (Lin et al. 2008) may also have contributed to the selection bias in our screens, as revealed by the isolation of multiple allelic mutants of several genes (Table 1). Nevertheless, the strategy used in this work still could be useful for similar studies in other bacterial pathogens without available genome sequences, particularly for isolating numerous defined mutants defective in LPS in order to investigate the functions of the molecules.

\section{MATERIALS AND METHODS}

\section{Bacteria strains and culture conditions.}

$R$. solanacearum Pss4 (phylotype I, biovar 3) (Lin et al. 2008) isolated from tomato in Taiwan was used as the wildtype strain in this study. Media used for this study were prepared as described previously (Boucher et al. 1985; Yang et al. 2013). The generation and growth evaluation of $R$. solanacearum Tn5 insertional mutants were performed as previously de-

\section{UDP-GIcNAC}
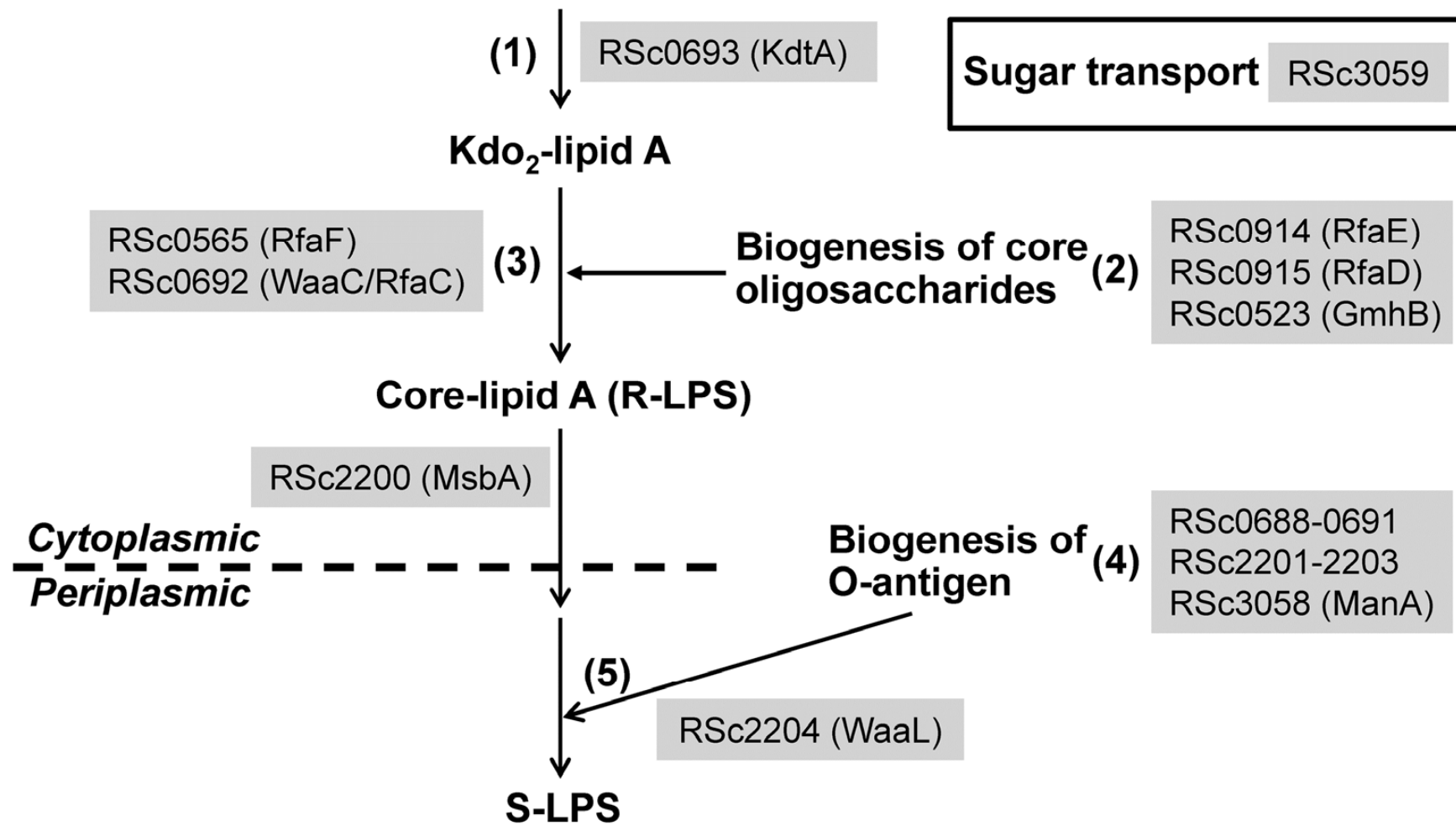

Fig. 4. Involvement of the identified proteins in key steps in the lipopolysaccharide (LPS) biosynthetic pathway in Ralstonia solanacearum. This pathway is modified from the Escherichia coli LPS biosynthesis pathway based on LPS profiling of the mutants identified in this study and the LPS biosynthesis-related functions of orthologs. 
scribed (Lin et al. 2008). For complementation tests, the lowcopy plasmid pUFR047 (De Feyter et al. 1993) was used to express a wild-type allele of the indicated genes. The coding sequences of the target genes and their upstream regions (488 bp for $R S c 2201,271$ bp for $R S c 2202$, and 494 bp for $R S c 2204$ and $R S c 2203+R S c 2204$ ) were amplified from $R$. solanacearum Pss 4 by PCR using primers designed based on sequences of strain GMI1000 (Supplementary Table S2). The PCR products were cloned into pUFR047 and verified for sequence accuracy. The resultant vectors were then introduced into the corresponding mutant strains by electroporation. After screening for gentamycin resistance, the presence and expression of the recombinant plasmids were confirmed by PCR genomic DNA and reverse-transcriptase PCR, respectively. Wild-type and mutant strains carrying empty pUFR047 were also generated and used as controls.

\section{Phage-resistant mutant screens.}

Two methods were performed for primary screens. In the first screening method, $100 \mu \mathrm{l}$ of bacterial culture grown for $48 \mathrm{~h}$ in 523 liquid medium was transferred into $500 \mu \mathrm{l}$ of 523 liquid medium containing kanamycin $(100 \mu \mathrm{g} / \mathrm{ml})$ and $\mathrm{RS} \varphi \mathrm{P} 29$ $\left(10^{7}\right.$ plaque-forming units per milliliter $\left.[\mathrm{PFU} / \mathrm{ml}]\right)$. Bacterial growth was assessed by absorbance at $600 \mathrm{~nm}$ after incubation at $28^{\circ} \mathrm{C}$ with shaking for $24 \mathrm{~h}$. The second method involved spotting bacterial cultures grown for $48 \mathrm{~h}$ onto soft-top agar, which was layered on CPG plates in the presence or absence of $\mathrm{RS} \varphi \mathrm{P} 29\left(10^{6} \mathrm{PFU} / \mathrm{ml}\right)$ using a 96-needle colony replicator. Bacterial growth on these plates was assessed after incubation at $28^{\circ} \mathrm{C}$ for $24 \mathrm{~h}$. For the secondary screens, putative RS $\varphi \mathrm{P} 29$ resistant mutants were grown in kanamycin-containing 523 liquid medium and diluted to $\mathrm{OD}_{600}=0.3$ in 523. Bacterial suspensions (10 $\mu \mathrm{l}$ each) were then spotted onto soft-top agar on CPG plates either containing or not containing RS $\varphi$ P29 $\left(10^{5} \mathrm{PFU} / \mathrm{ml}\right)$ and bacterial growth was assessed after incubation at $28^{\circ} \mathrm{C}$ for $24 \mathrm{~h}$. Mutants that grew on CPG containing $\mathrm{RS} \varphi \mathrm{P} 29$ were identified as $\mathrm{RS} \varphi \mathrm{P} 29$-resistant mutants and subjected to further analyses.

\section{Determination and bioinformatic analysis of the disrupted loci.}

Bacterial genomic DNA was isolated following a method described by Chen and Kuo (1993). To determine the genomic sequence flanking Tn5, TAIL-PCR tests were performed successively with three Tn5 sequence-specific primers paired to one of the arbitrary degenerate primers (Liu et al. 1995). The parameters of these PCR reactions (Supplementary Tables S3 and S4) were established by modifying a method described by Qian and associates (2005). The amplified fragments were cloned into pCRII-TOPO (Invitrogen, Carlsbad, CA, U.S.A.), sequenced, and compared with the $R$. solanacearum GMI1000 sequence by BLASTN at the National Center for Biotechnology Information (NCBI) database. The putative Tn5-inserted loci were then confirmed by genomic PCR using primers specific for Tn5 and the target gene. Possible functions and conserved domains of the identified loci were predicted at the NCBI and KEGG databases. The sequence homologies of individual genes to orthologs in E. coli and other bacteria were analyzed by PSI-BLAST/PHI-BLAST algorithm.

\section{Analysis of LPS biogenesis.}

LPS was prepared and analyzed as previously described (Yang et al. 2013). Briefly, total LPS extracted from bacterial cells $\left(\mathrm{OD}_{600}=0.4\right)$ was treated with DNase, RNase, and proteinase $\mathrm{K}$ and then fractionated on regular 4 to $12 \%$ SDSpolyacrylamide gels (Tsai and Frasch 1982) or 16\% tricine-
SDS-polyacrylamide (Schägger 2006) gels, followed by silver staining.

\section{Pathogenesis assays.}

Leaves of $N$. benthamiana and $N$. tabacum 'Wisconsin 38' were infiltrated with bacterial suspensions at $\mathrm{OD}_{600}=0.3$ (approximately $10^{8} \mathrm{CFU} / \mathrm{ml}$ ) and 1.0 , respectively. The plants were incubated at room temperature. Development of symptoms in $N$. benthamiana and the HR in N. tabacum were recorded periodically. To assess in planta bacterial proliferation, a method modified from Solé and associates (2012) was used. Bacterial suspensions at approximately $10^{4}$ to $10^{5} \mathrm{CFU} / \mathrm{ml}$ were used to infiltrate $N$. benthamiana leaves. For each trial, three discs $(1 \mathrm{~cm}$ in diameter) of the infiltrated leaves were recovered from each of two test plants $24 \mathrm{~h}$ after infiltration. Two discs were combined into one sample, weighed, and completely ground in $1 \mathrm{ml}$ of sterile distilled water. The resultant suspensions were serially diluted 10 -fold and $10 \mu \mathrm{l}$ of each diluted sample was spotted on SM1 medium plates in replicates to count CFU. Virulence tests on 3-week-old susceptible L390 tomato were performed by soil drench with bacteria at $\mathrm{OD}_{600}=0.3$ without root severing, as described previously (Lin et al. 2008).

\section{ACKNOWLEDGMENTS}

This work was supported by grants (NSC 98-2321-B-002-020-MY3 and NSC 101-2313-B-002-055) from the National Science Council of Taiwan, Republic of China, to C.-P. Cheng. This work has been carried out in compliance with local regulations on genetic recombination experimentation. We thank G. Gussin for critical review and helpful discussion; and the excellent technical assistance of Technology Commons, College of Life Science, National Taiwan University, for the ultracentrifuges (TCXLS06), the plant greenhouse facility (TCX-E), and quantitative reversetranscriptase PCR analyses.

\section{LITERATURE CITED}

Boucher, C. A., Barberis, A. J., and Demery, D. A. 1985. Transposon mutagenesis of Pseudomonas solanacearum: Isolation of Tn5-induced avirulent mutants. J. Gen. Microbiol. 131:2449-2457.

Chen, W. P., and Kuo, T. T. 1993. A simple and rapid method for the preparation of gram-negative bacterial genomic DNA. Nucleic Acids Res. $21: 2260$.

Chng, S. S., Gronenberg, L. S., and Kahne, D. 2010a. Proteins required for lipopolysaccharide assembly in Escherichia coli form a trans-envelope complex. Biochemistry 49:4565-4567.

Chng, S. S., Ruiz, N., Chimalakonda, G., Silhavy, T. J., and Kahne, D. 2010b. Characterization of the two-protein complex in Escherichia coli responsible for lipopolysaccharide assembly at the outer membrane. Proc. Natl. Acad. Sci. U.S.A. 107:5363-5368.

Collins, L. V., Attridge, S., and Hackett, J. 1991. Mutations at $r f c$ or $p m i$ attenuate Salmonella typhimurium virulence for mice. Infect. Immun. 59:1079-1085.

De Feyter, R., Yang, Y., and Gabriel, D. W. 1993. Gene-for-genes interactions between cotton $R$ genes and Xanthomonas campestris pv. malvacearum avr genes. Mol. Plant-Microbe Interact. 6:225-237.

Denny, T. P. 2006. Plant pathogenic Ralstonia species. Pages 573-644 in: Plant-Associated Bacteria. S. S. Gnanamanickam, ed. Springer Publishing, Dordrecht, The Netherlands.

Denny, T. P., and Hayward, A. C. 2001. Ralstonia solanacearum. Pages 151-173 in: Laboratory Guide for Identification of Plant Pathogenic Bacteria, 3rd ed. N. W. Schaad, J. B. Jones, and W. Chun, eds. American Phytopathological Society Press, St. Paul, MN, U.S.A.

Dow, M., Newman, M. A., and von Roepenack, E. 2000. The induction and modulation of plant defense responses by bacterial lipopolysaccharides. Annu. Rev. Phytopathol. 38:241-261.

Graham, T. L., Sequeira, L., and Huang, T. S. 1977. Bacterial lipopolysaccharides as inducers of disease resistance in tobacco. Appl. Environ. Microbiol. 34:424-432.

Hendrick, C. A., and Sequeira, L. 1984. Lipopolysaccharide-defective mutants of the wilt pathogen Pseudomonas solanacearum. Appl. Environ. Microbiol. 48:94-101.

Heller, K., and Braun, V. 1982. Polymannose O-antigens of Escherichia 
coli, the binding sites for the reversible adsorption of bacteriophage T5+ via the L-shaped tail fibers. J. Virol. 41:222-227.

Kim, S. H., Ahn, S. H., Lee, J. H., Lee, E. M., Kim, N. H., Park, K. J., and Kong, I. S., 2003. Genetic analysis of phosphomannomutase/phosphoglucomutase from Vibrio furnissii and characterization of its role in virulence. Arch. Microbiol. 180:240-250.

Kocharova, N. A., Knirel, Y. A., Shashkov, A. S., Nifant'ev N. E., Kochetkov, N. K., Varbanets, L. D., Moskalenko, N. V., Brovarskaya, O. S., Muras, V. A., and Young, J. M. 1993. Studies of O-specific polysaccharide chains of Pseudomonas solanacearum lipopolysaccharides consisting of structurally different repeating units. Carbohydr. Res. 250:275-287.

Lin, Y. M., Chou, I. C., Wang, J. F., Ho, F. I., Chu, Y. J., Huang, P. C., Lu, D. K., Shen, H. L., Elbaz, M., Huang, S. M., and Cheng, C. P. 2008. Transposon mutagenesis reveals differential pathogenesis of Ralstonia solanacearum on tomato and Arabidopsis. Mol. Plant-Microbe Interact. 21:1261-1270.

Liu, Y. G., Mitsukawa, N., Oosumi, T., and Whittier, R. F. 1995. Efficient isolation and mapping of Arabidopsis thaliana T-DNA insert junctions by thermal asymmetric interlaced PCR. Plant J. 8:457-463.

Michel, A., Clermont, O., Denamur, E., and Tenaillon, O. 2010. Bacteriophage PhiX174's ecological niche and the flexibility of its Escherichia coli lipopolysaccharide receptor. Appl. Environ. Microbiol. 76:73107313.

Newman, M. A., Dow, J. M., Molinaro, A., and Parrilli, M. 2007. Priming, induction and modulation of plant defence responses by bacterial lipopolysaccharides. J. Endotoxin Res. 13:69-84.

Ormeno-Orrillo, E., Rosenblueth, M., Luyten, E., Vanderleyden, J., and Martinez-Romero, E. 2008. Mutations in lipopolysaccharide biosynthetic genes impair maize rhizosphere and root colonization of Rhizobium tropici CIAT899. Environ. Microbiol. 10:1271-1284.

Paixão, T. A., Roux, C. M., Hartigh, A. B., Sumathi S. W., Dandekar, S., Santos R. L., and Tsolis, R. M., 2009. Establishment of systemic Brucella melitensis infection through the digestive tract requires urease, the type IV secretion system, and lipopolysaccharide O-antigen. Infect. Immun. 77:4197-4208.

Prehm, P., Jann, B., Jann, K., Schmidt, G., and Stirm, S. 1976. On a bacteriophage $\mathrm{T} 3$ and $\mathrm{T} 4$ receptor region within the cell wall lipopolysaccharide of Escherichia coli. Br. J. Mol. Biol. 101:277-281.

Qian, W., Jia, Y., Ren, S. X., He, Y. Q., Feng, J. X., Lu, L. F., Sun, Q., Ying, G., Tang, D. J., Tang, H., Wu, W., Hao, P., Wang, L., Jiang, B. L., Zeng, S., Gu, W. Y., Lu, G., Rong, L., Tian, Y., Yao, Z., Fu, G., Chen, B., Fang, R., Qiang, B., Chen, Z., Zhao, G. P., Tang, J. L., and He, C. 2005. Comparative and functional genomic analyses of the pathogenicity of phytopathogen Xanthomonas campestris pv. campestris. Genome Res. 15:757-767.

Qimron, U., Marintcheva, B., Tabor, S., and Richardson, C. C. 2006. Genomewide screens for Escherichia coli genes affecting growth of T7 bacteriophage. Proc. Natl. Acad. Sci. U.S.A. 103:19039-19044.

Raetz, C. R., and Whitfield, C. 2002. Lipopolysaccharide endotoxins.
Annu. Rev. Biochem. 71:635-700.

Schägger, H. 2006. Tricine-SDS-PAGE. Nat. Protocols 1:16-22.

Solé, M., Popa, C., Mith O., Sohn, K. H., Jones, J. D. G., Deslandes, L., and Valls, M. 2012. The awr gene family encodes a novel class of Ralstonia solanacearum type III effectors displaying virulence and avirulence activities. Mol. Plant-Microbe Interact. 25:941-953.

Sperandeo, P., Deho, G., and Polissi, A. 2009. The lipopolysaccharide transport system of gram-negative bacteria. Biochim. Biophys. Acta 1791:594-602.

Titarenko, E., Lopez-Solanilla, E., Garcia-Olmedo, F., and RodriguezPalenzuela, P. 1997. Mutants of Ralstonia (Pseudomonas) solanacearum sensitive to antimicrobial peptides are altered in their lipopolysaccharide structure and are avirulent in tobacco. J. Bacteriol. 179:6699-6704.

Tsai, C. M., and Frasch, C. E. 1982. A sensitive silver stain for detecting lipopolysaccharides in polyacrylamide gels. Anal. Biochem. 119:115-119.

Varbanets, L. D., Vasil'ev, V. N., and Brovarskaia, O.S. 2003. Characterization of lipopolysaccharides from Ralstonia solanacearum. Mikrobiologiia $72: 19-25$

Wang, X., and Quinn, P. J. 2010. Lipopolysaccharide: Biosynthetic pathway and structure modification. Prog. Lipid Res. 49:97-107.

Whatley, M. H., Hunter, N., Cantrell, M. A., Hendrick, C., Keegstra, K., and Sequeira, L. 1980. Lipopolysaccharide composition of the wilt pathogen, Pseudomonas solanacearum: Correlation with the hypersensitive response in tobacco. Plant Physiol. 65:557-559.

Wilkinson, S. G. 1996. Bacterial lipopolysaccharides-themes and variations. Prog. Lipid Res. 3:283-343.

Yang, W. C., Lin, Y. M., Cheng, Y. S., and Cheng, C. P. 2013. Ralstonia solanacearum RSc0411 (lptC) is a determinant for full virulence and has a strain-specific novel function in the T3SS activity. Microbiology 159:1136-1148.

Zdorovenko, E. L., Vinogradov, E., Wydra, K., Lindner, B., and Knirel, Y. A. 2008. Structure of the oligosaccharide chain of the SR-type lipopolysaccharide of Ralstonia solanacearum Toudk-2. Biomacromolecules 9:2215-2220

\section{AUTHOR-RECOMMENDED INTERNET RESOURCES}

European Molecular Biology Laboratory-European Bioinformatics Institute PSI-BLAST database: www.ebi.ac.uk/Tools/blastpgp/index.html

Institut National de la Recherche Agronomique Ralstonia solanacearum webpage: bioinfo.genopole-toulouse.prd.fr/annotation/iANT/bacteria/ralsto

Kyoto Encyclopedia of Genes and Genomes (KEGG) database: www.genome.jp/kegg

National Center for Biotechnology Information database: www.ncbi.nlm.nih.gov 